\title{
High expression of miR-363 predicts poor prognosis and guides treatment selection in acute myeloid leukemia
}

Huihui Zhang ${ }^{1,2 \dagger}$, Ninghan Zhang ${ }^{1,2+}$, Rong Wang ${ }^{1,2 \dagger}$, Tingting Shao ${ }^{1}$, Yuan Feng ${ }^{1}$, Yao Yao ${ }^{1,2}$, Qingyun Wu ${ }^{1,2}$,

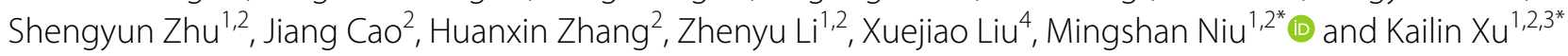

\begin{abstract}
Background: Acute myeloid leukemia (AML) is a highly heterogeneous malignancy with various outcomes, and therefore needs better risk stratification tools to help select optimal therapeutic options.

Methods: In this study, we identify miRNAs that could predict clinical outcome in a heterogeneous AML population using TCGA dataset.

Results: We found that MiR-363 is a novel prognostic factor in AML patients undergoing chemotherapy. In multivariable analyses, high miR-363 remained predictive for shorter $O S(H R=2.349, P=0.012)$ and $E F S(H R=2.082, P=0.001)$ independent of other well-known prognostic factors. More importantly, allogeneic hematopoietic stem cell transplantation (allo-HSCT) overcame the adverse outcomes related to high miR-363 expression. In gene expression profiling, high miR-363 expression was positively correlated with the amounts of leukemogenic transcription factors, including Myb, RUNX3, GATA3, IKZF3, ETS1 and MLLT3. Notably, we found that the in silico predicted target genes (EZH2, KLF6 and PTEN) of miR-363 were downregulated in association with high miR-363 expression.

Conclusions: In summary, miR-363 expression may help identify patients in need of strategies to select the optimal therapy between chemotherapeutic and allo-HCST regimens. AML patients with high miR-363 expression may be highly recommended for early allo-HSCT regimen.
\end{abstract}

Keywords: Mir-363, Acute myeloid leukemia, Clinical outcome, Chemotherapy, Allo-HSCT

\section{Background}

Acute myeloid leukemia (AML), the most frequent form of acute leukemia in adults, is caused by a rapid clonal proliferation of neoplastic myeloblasts [1]. Patients with AML manifest complex and heterogeneous outcomes after receiving different treatments. Conventional cytotoxic treatment with chemotherapeutics is the first-line therapy in AML [2]. High-risk patients could receive effectively antileukemic action and potential cure after accepting allogeneic hematopoietic stem

\footnotetext{
*Correspondence: msniu24@126.com; lihmd@163.com

${ }^{\dagger}$ Huihui Zhang, Ninghan Zhang and Rong Wang contributed equally to this work

${ }^{1}$ Blood Diseases Institute, Xuzhou Medical University, Xuzhou, Jiangsu, China

Full list of author information is available at the end of the article
}

cell transplantation (allo-HSCT). The variation in AML patient prognosis is related to various inherent factors, including cytogenetics and genetic alterations. Somatic mutations in NPM1, CEBPA, FLT3, IDH1, IDH2 and TET2 are associated with outcomes of patients and served as prognostic markers in AML [3]. Despite the molecular mechanisms of leukemogenesis are well known, most AML patients are not cured. Notably, the currently available risk stratification systems are not completely accurate. Therefore, novel prognostic markers are needed to improve AML risk classification and select optimal therapeutic schedule.

MicroRNAs (miRNAs) represent short noncoding RNAs, which hybridize to target mRNAs with high specificity and decrease protein levels through translation inhibition [4]. Dysregulation of miRNAs expression in

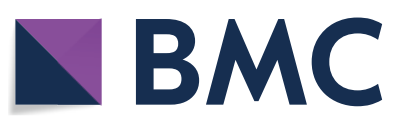

(c) The Author(s) 2019. This article is distributed under the terms of the Creative Commons Attribution 4.0 International License (http://creativecommons.org/licenses/by/4.0/), which permits unrestricted use, distribution, and reproduction in any medium, provided you give appropriate credit to the original author(s) and the source, provide a link to the Creative Commons license, and indicate if changes were made. The Creative Commons Public Domain Dedication waiver (http://creativecommons.org/ publicdomain/zero/1.0/) applies to the data made available in this article, unless otherwise stated. 
AML can affect cell proliferation, survival and hematopoietic differentiation [5]. More importantly, abnormal expression of miRNAs is related to clinical outcomes of AML patients. For instance, high miR-181a level has been confirmed to predict favorable survival in cytogenetically normal AML cases [6]. Patients with high miR-212 level tend to have better outcome independently of cytogenetic subtype [7]. Moreover, high miR-3151 expression is associated with worse overall survival and diseasefree survival in patients cytogenetically normal AML [8]. However, the majority of previous studies did not distinguish the various effects of chemotherapy and allo-HSCT treatment on the therapeutic outcome. As is well known, the prognostic impact of a marker is treatment-dependent in AML. Consequently, miRNAs may have different prognostic effects in chemotherapy and allo-HSCT treatment, respectively.

In this study, we identify miR-363 that could predict clinical outcome in a heterogeneous AML population using genome-wide screening. The prognostic role of miR-363 is independent of known potent clinical and molecular predictors. The miR-363 expression contributed to risk classification in AML patients undergoing chemotherapy. We also evaluated whether allo-HCST could overcome the poor prognostic effects of high miR363 level in the same cohort. In order to evaluate biological insights of miR-363, we performed genome-wide gene and miRNA expression analyses.

\section{Materials and methods}

\section{Patients}

We studied a total of 162 patients with newly diagnosed AML according to the WHO classification. The RNASeq expression data of these AML patients were provided by The Cancer Genome Atlas (TCGA) [9]. This study has been approved by Human Studies Committee of the Washington University. Patients with AML were included in a single center's tissue protocol and followed NCCN guidelines to receive treatment. Patients with unfavorable risk underwent allo-HSCT if they were medically fit for the risks of transplantation, and if a suitably matched donor was available. In this cohort, 90 patients were only treated by chemotherapy and another 72 patients accepted both chemotherapy and allo-HSCT. All clinical data are available on the TCGA website.

\section{Gene-expression profiling}

Of the 162 patients, only 155 had both microRNA and mRNA expression data. For mRNA-seq data, genes expressed at or below a noise threshold of RPKM (Reads per kilobase per million mapped reads) $\leq 0.2$ in at least $75 \%$ of samples were removed. For miRNA-seq data, read counts were normalized to RPM (Reads per million reads). The expression data were $\log 2$ transformed before analysis. The gene/microRNA expression signatures were derived by Spearman correlation analysis (BenjaminiHochberg adjusted $P$ value $<0.01$ ). Finally, gene rows were reordered using hierarchical clustering analysis. The miRBase Targets Version 7 and Targetscan Release 7.1 were employed to predict the targets of miR-363. Gene Ontology enrichment assessment of genes in miR363 related signature was conducted with the Database for Annotation, Visualization, and Integrated Discovery (DAVID).

\section{Statistical analysis}

A comparison of baseline characteristics between patients with high and low miRNA expression patients was conducted. The median miR-363 level was used to identify patients with low and high miRNA expression, respectively. Mann-Whitney $U$ test was performed to test relations between two continuous variables. Fisher's exact and Chi square tests were determined for categorical variables. Overall survival (OS) was the time from patient diagnosis to death at the final follow-up. Eventfree survival (EFS) was the time from patient diagnosis to adverse events, including relapse and death. KaplanMeier method was performed to evaluate OS and EFS distributions and the log-rank test was employed to compare survival curves.

Univariable Cox proportional hazards models were constructed for assessing correlations of miR-363 expressions with OS and EFS, respectively. We establish multivariable Cox proportional hazards models to identify factors affecting OS and EFS. The factors included in the evaluation model contained miR-363 expression levels, FLT3-ITD, NPM1, DNMT3A, RUNX1, TP53, TET2, MLL-PTD, IDH1/IDH2 and NRAS/KRAS mutation statuses, and WBC involvement. Factors showing significance with $\alpha=0.20$ in univariable analysis were entered into limited backward selection to generate multivariable models. Variables remaining in the final models were significant at $\alpha=0.05$. The $\mathrm{R}$ software 3.1.5, GraphPad Prism and SPSS were used for statistical analysis, with $P<0.05$ indicating statistical significance.

\section{Results}

\section{Association of miR-363 level with clinico-molecular properties}

The patients were divided into two groups, chemotherapy and allo-HSCT groups. Subsequently, each group was subdivided into two groups in accordance with the median of miR-363. The relationship between clinicalgenetic characteristics and miR-363 expression is shown in Table 1. In patients who underwent chemotherapy, cases with high miR-363 levels showed higher relapse rate 
Table 1 Comparison of clinical and molecular characteristics with miR-363 expression in AML patients

\begin{tabular}{|c|c|c|c|c|c|c|}
\hline \multirow[t]{2}{*}{ Characteristic } & \multicolumn{3}{|l|}{ Chemotherapy group } & \multicolumn{3}{|l|}{ Allo-HSCT group } \\
\hline & High miR-363 $(n=45)$ & Low miR-363 $(n=45)$ & $P$ & High miR-363 $(n=36)$ & Low miR-363 $(n=36)$ & $P$ \\
\hline Age/years, median & $68(33-88)$ & $62(22-77)$ & 0.005 & $52(18-65)$ & $51(21-72)$ & 0.585 \\
\hline Age group, $\mathrm{n}(\%)$, years & & & 0.006 & & & 0.793 \\
\hline$<60$ & $8(17.8)$ & $21(46.7)$ & & $25(69.4)$ & $27(75.0)$ & \\
\hline$\geq 60$ & $37(82.2)$ & $24(53.3)$ & & $11(30.6)$ & $9(25.0)$ & \\
\hline Gender, n (\%) & & & 1.000 & & & 0.634 \\
\hline Male & $25(55.6)$ & $25(55.6)$ & & $22(55.6)$ & $19(52.8)$ & \\
\hline Female & $20(44.4)$ & $20(44.4)$ & & $14(38.9)$ & $17(47.2)$ & \\
\hline WBC, $\times 10^{9} / L$, median & $8.3(0.7-171.9)$ & $39.8(2.5-298.4)$ & 0.001 & $11.4(0.6-77.3)$ & $35.8(1.2-223.8)$ & 0.001 \\
\hline BM blast (\%), median & $71(30-98)$ & 73 (32-99) & 0.580 & $67.5(30-95)$ & $71.5(39-100)$ & 0.156 \\
\hline PB blast (\%), median & $16(0-91)$ & $52(0-98)$ & 0.007 & $33.5(0-90)$ & $56(8-96)$ & 0.008 \\
\hline \multicolumn{7}{|l|}{ FAB subtypes, n (\%) } \\
\hline MO & $5(11.1)$ & $3(6.7)$ & 0.714 & $7(19.4)$ & $2(5.6)$ & 0.151 \\
\hline M1 & $9(20)$ & $11(24.4)$ & 0.800 & $10(37.8)$ & $13(36.1)$ & 0.614 \\
\hline M2 & $10(22.2)$ & $11(24.4)$ & 1.000 & $9(25.0)$ & $10(27.8)$ & 1.000 \\
\hline M4 & $10(22.2)$ & $14(31.1)$ & 0.475 & $5(13.9)$ & $9(25.0)$ & 0.372 \\
\hline M5 & $8(17.8)$ & $5(11.1)$ & 0.550 & $3(8.3)$ & $1(2.8)$ & 0.614 \\
\hline M6 & $2(4.4)$ & $1(2.2)$ & 1.000 & $1(2.8)$ & 0 & 1.000 \\
\hline M7 & $1(2.2)$ & $0(0)$ & 1.000 & $1(2.8)$ & 0 & 1.000 \\
\hline Others & $1(2.2)$ & $0(0)$ & 1.000 & 0 & $1(2.8)$ & 1.000 \\
\hline \multicolumn{7}{|l|}{ Karyotype, n (\%) } \\
\hline Normal & $20(44.4)$ & $24(53.3)$ & 0.527 & $16(44.4)$ & $18(50.0)$ & 0.814 \\
\hline Complex & $11(24.4)$ & $1(2.2)$ & 0.004 & $11(30.6)$ & $1(2.8)$ & 0.003 \\
\hline 8 Trisomy & 0 & $2(4.4)$ & 0.494 & $2(5.6)$ & $5(13.9)$ & 0.429 \\
\hline CBF $\beta-M Y H 11$ & 0 & $7(15.6)$ & 0.012 & 0 & $5(13.9)$ & 0.054 \\
\hline $11 \mathrm{q} 23 / \mathrm{MLL}$ & $4(8.9)$ & $1(2.2)$ & 0.361 & $2(5.6)$ & $1(2.8)$ & 1.000 \\
\hline$-7 / 7 q-$ & $2(4.4)$ & $1(2.2)$ & 1.000 & $1(2.8)$ & $1(2.8)$ & 1.000 \\
\hline BCR-ABL1 & $1(2.2)$ & 0 & 1.000 & $1(2.8)$ & $1(2.8)$ & 1.000 \\
\hline RUNX1-RUNX1T & $1(2.2)$ & $5(11.1)$ & 0.203 & 0 & $1(2.8)$ & 1.000 \\
\hline Others & $6(13.3)$ & $4(8.9)$ & 0.739 & $3(8.3)$ & $3(8.3)$ & 1.000 \\
\hline \multicolumn{7}{|l|}{ Risk, n (\%) } \\
\hline Good & $1(2.2)$ & $12(26.7)$ & 0.002 & 0 & $6(16.7)$ & 0.025 \\
\hline Intermediate & $25(55.6)$ & $25(55.6)$ & 1.000 & $19(52.8)$ & $22(61.1)$ & 0.634 \\
\hline Poor & $18(40.0)$ & $7(15.6)$ & 0.018 & $17(47.2)$ & $7(19.4)$ & 0.023 \\
\hline Others & $1(2.2)$ & $1(2.2)$ & 1.000 & 0 & $1(2.8)$ & 1.000 \\
\hline FLT3-ITD, n (\%) & & & 0.784 & & & 0.045 \\
\hline Presence & $7(15.6)$ & $9(20.0)$ & & $4(11.1)$ & $12(33.3)$ & \\
\hline Absence & $38(84.4)$ & $36(80.0)$ & & $32(88.9)$ & $24(66.7)$ & \\
\hline NPM1, n (\%) & & & 0.023 & & & 0.430 \\
\hline Mutation & $9(20.0)$ & $20(44.4)$ & & $8(22.2)$ & $12(33.3)$ & \\
\hline Wild type & $36(80.0)$ & 25 (55.6) & & $28(77.8)$ & $24(66.7)$ & \\
\hline \multicolumn{7}{|l|}{ CEBPA, n (\%) } \\
\hline Single mutation & $1(2.2)$ & $2(4.4)$ & 1.000 & 0 & $5(13.9)$ & 0.054 \\
\hline Double mutation & 0 & 0 & & $1(2.8)$ & $2(5.6)$ & 1.000 \\
\hline Wild type & $44(97.8)$ & $43(95.6)$ & 1.000 & $35(97.2)$ & $29(80.6)$ & 0.055 \\
\hline DNMT3A, n (\%) & & & 1000 & & & 0.786 \\
\hline Mutation & $13(28.9)$ & $12(26.7)$ & & $10(27.8)$ & $8(22.2)$ & \\
\hline Wild type & $32(71.1)$ & 33 (73.3) & & $26(72.2)$ & $28(7.8)$ & \\
\hline $\mathrm{IDH} 1 / \mathrm{IDH} 2, \mathrm{n}(\%)$ & & & 0.167 & & & 0.415 \\
\hline
\end{tabular}


Table 1 (continued)

\begin{tabular}{|c|c|c|c|c|c|c|}
\hline \multirow[t]{2}{*}{ Characteristic } & \multicolumn{3}{|l|}{ Chemotherapy group } & \multicolumn{3}{|l|}{ Allo-HSCT group } \\
\hline & High miR-363 $(n=45)$ & Low miR-363 $(n=45)$ & $P$ & High miR-363 $(n=36)$ & Low miR-363 $(n=36)$ & $P$ \\
\hline Mutation & $5(11.1)$ & $11(24.4)$ & & $11(30.6)$ & $7(19.4)$ & \\
\hline Wild type & $40(88.9)$ & $34(75.6)$ & & $25(69.4)$ & $29(80.6)$ & \\
\hline RUNX1, n (\%) & & & 0.714 & & & 0.260 \\
\hline Mutation & $5(11.1)$ & $3(6.7)$ & & $6(16.7)$ & $2(5.6)$ & \\
\hline Wild type & 40 (88.9) & $42(93.3)$ & & $30(83.3)$ & $34(94.4)$ & \\
\hline MLL-PTD, n (\%) & & & 1.000 & & & 0.614 \\
\hline Presence & $2(4.4)$ & $3(6.7)$ & & $3(8.3)$ & $1(2.8)$ & \\
\hline Absence & $43(95.6)$ & $42(93.7)$ & & $33(91.7)$ & $35(97.2)$ & \\
\hline NRAS/KRAS, n (\%) & & & 1.000 & & & 1.000 \\
\hline Mutation & $6(13.3)$ & $7(15.6)$ & & $4(11.1)$ & $3(8.3)$ & \\
\hline Wild type & 39 (86.7) & $38(84.4)$ & & $32(88.9)$ & $33(91.7)$ & \\
\hline TET2, n (\%) & & & 0.118 & & & 0.614 \\
\hline Mutation & $9(20.0)$ & $3(6.7)$ & & $1(2.8)$ & $3(8.3)$ & \\
\hline Wild type & $36(80.0)$ & $42(93.7)$ & & $35(97.2)$ & $33(91.7)$ & \\
\hline TP53, n (\%) & & & 0.000 & & & 0.115 \\
\hline Mutation & $11(24.4)$ & 0 & & $4(11.1)$ & 0 & \\
\hline Wild type & $34(75.6)$ & $45(100.0)$ & & $32(88.9)$ & $36(100.0)$ & \\
\hline Relapse, n (\%) & & & 0.001 & & & 0.474 \\
\hline Yes & $42(93.3)$ & $28(62.2)$ & & $23(63.9)$ & $19(47.2)$ & \\
\hline No & $3(6.7)$ & 17 (37.8) & & $13(36.1)$ & $17(52.8)$ & \\
\hline
\end{tabular}

Mann-Whitney test was used for continuous variables. Chi square tests were used for categorical variables

$W B C$ white blood cell, $B M$ bone marrow, $P B$ peripheral blood, $F A B$ French-American-British classification

$(P=0.001)$, and lower WBC count $(P=0.001)$ and circulating blast amounts $(P=0.007)$ at initial diagnosis in comparison with those expressing low miR-363 amounts. Patients with elevated miR-363 expression comprised less cases with favorable risk $(P=0.002)$, but more with poor risk of AML $(P=0.018)$. Furthermore, patients with high miR-363 expression included $92 \%$ of all cases with complex karyotypes and all cases with TP53 gene mutation. Meanwhile, Low miR-363 expressers encompassed all cases with the CBF $\beta-$ MYH11 fusion gene and $69 \%$ of all cases with NPM1 gene mutation.

Prognostic value of miR-363 expression in patients treated with chemotherapy or allo-HSCT

We performed genome-wide screening of miRNAs in AML cases in order to acquire prognostic marker to improve the classification of AML. MiR-363 was identified as a new prognostic marker for chemotherapy in AML patients. In order to evaluate survival of patients, we employed the Kaplan-Meier method and log-rank test. The expression level distribution of miR-363 was shown in Fig. 1a. In the chemotherapy group, cases highly expressing miR-363 showed reduced OS $(\mathrm{HR}=2.28$, $P=0.0004)$ and EFS $(\mathrm{HR}=2.14, P=0.0012)$ in comparison with low expressers (Fig. 1b). We further performed a survival analysis in the good/intermediate group, patients with high miR-363 expression had significantly shorter OS $(P=0.0009)$ and EFS $(P=0.0019)$ compared with patients with low miR-363 expression (Fig. 1c). However, miR-363 expression level was not associated with outcome in AML patients treated with allo-HCST (Fig. 1d). These data suggested that high expression of miR-363 was a poor prognostic factor in AML patients treated with chemotherapy.

\section{MiR-363 is associated with clinical outcome in AML}

Univariate and multivariate cox analyses were performed to assess whether miR-363 level is an independent predictor of survival in AML. Univariate analysis (Table 2) showed that high miR-363 had an adverse prognostic value for predicting $\mathrm{OS}(\mathrm{HR}=2.389, P<0.001)$ and $\mathrm{EFS}$ $(\mathrm{HR}=2.224, P=0.001)$ in cases administered chemotherapy. In multivariable analysis, miR-363 and multiple demonstrated prognostic factors were assessed (Table 2). High miR-363 remained an independent predictor of shorter OS $(\mathrm{HR}=2.349,95 \% \mathrm{CI} 1.305-4.229, P=0.012)$ and EFS $(\mathrm{HR}=2.082,95 \% \mathrm{CI} 1.172-3.699, P=0.001)$.

In patients receiving allo-HSCT, univariate analysis showed that adverse OS in patients with TP53-mutant. However, miR-363 expression status was not associated 

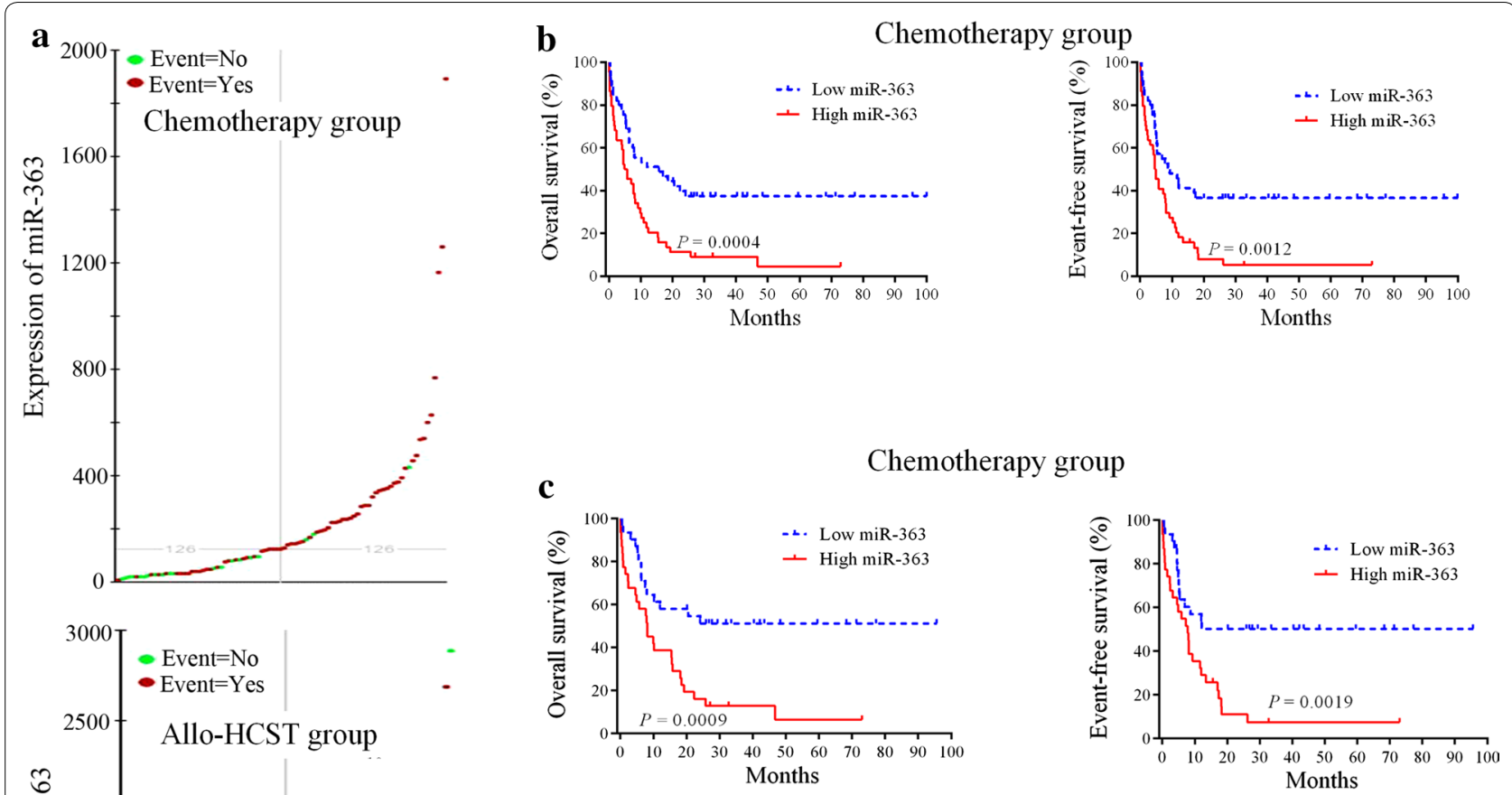

d

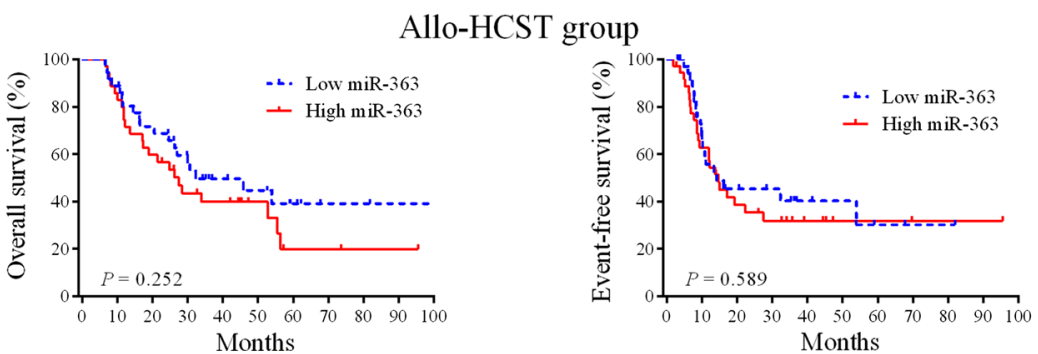

Fig. 1 Kaplan-Meier survival curves based on miR-363 expression. a The expression level distribution of miR-363. b Cases highly expressing miR-363 showed markedly shorter OS and EFS in the chemotherapy group $(n=90)$. c Patients with high miR-363 expression had poor OS and EFS in the chemotherapy group. $\mathbf{d}$ Effect of miR-363 levels on OS and EFS in cases administered allo-HSCT $(n=72)$

with OS and EFS in the allo-HSCT group (Table 3). Multivariable analysis revealed that TP53 and FLT3-ITD mutations independently predict adverse OS $(P=0.002$ and $P=0.049$, respectively). The miR-363 expression status did not persist as OS and EFS predictors in multivariable analysis.

\section{Allo-HSCT overcomes the adverse prognostic role of miR-363 expression}

Next, we investigated whether allo-HSCT could overcome the adverse outcomes of miR-363 expression. The 162 patients were divided into 2 groups according to the median level of miR-363. In the high miR-363 group, cases administered allo-HSCT showed markedly improved OS $(\mathrm{HR}=0.361,95 \%$ CI $0.225-0.588$,
$P<0.0001)$ and EFS $(\mathrm{HR}=0.447,95 \%$ CI $0.287-0.751$, $P=0.002)$ in comparison with cases administered chemotherapy (Fig. 2a). In patients with lower expression of miR-363, no marked differences in OS $(P=0.127)$ and EFS $(P=0.226)$ were found between the chemotherapy and allo-HSCT groups (Fig. 2b). These results suggested that miR-363 may be considered as a prognostic marker for the detection of patients requiring optimal therapeutic schedules.

\section{Biological insight of miR-363 expression in AML}

To further investigate the biological function of miR363 , gene expression signature associated with miRNA expression was determined in AML cases. We observed that the levels of 178 genes were strongly associated with 
Table 2 Univariate and multivariate analyses in patients treated with chemotherapy

\begin{tabular}{|c|c|c|c|c|}
\hline \multirow[t]{2}{*}{ Variables } & \multicolumn{2}{|l|}{ EFS } & \multicolumn{2}{|l|}{ os } \\
\hline & HR $(95 \% \mathrm{Cl})$ & $P$-value & HR $(95 \% \mathrm{Cl})$ & $P$-value \\
\hline \multicolumn{5}{|l|}{ Univariate analyses } \\
\hline MiR-363 (high vs. low) & $2.224(1.369-3.612)$ & 0.001 & $2.389(1.468-3.887)$ & 0.000 \\
\hline WBC $\left(\geq 20\right.$ vs. $\left.<20 \times 10^{9} / L\right)$ & $1.015(0.633-1.627)$ & 0.952 & $0.980(0.611-1.571)$ & 0.932 \\
\hline FLT3-ITD (positive vs. negative) & $1.095(0.587-2.040)$ & 0.776 & $1.049(0.563-1.956)$ & 0.880 \\
\hline NPM1 (mutated vs. wild) & $1.050(0.633-1.741)$ & 0.850 & $0.965(0.582-1.599)$ & 0.890 \\
\hline DNMT3A (mutated vs. wild) & $1.301(0.774-2.185)$ & 0.320 & $1.299(0.775-2.179)$ & 0.321 \\
\hline RUNX1 (mutated vs. wild) & $1.502(0.717-3.147)$ & 0.281 & $1.591(0.759-3.335)$ & 0.219 \\
\hline TP53 (mutated vs. wild) & $3.011(1.539-5.892)$ & 0.001 & $2.898(1.487-5.649)$ & 0.002 \\
\hline TET2 (mutated vs. wild) & $0.778(0.372-1.625)$ & 0.504 & $0.686(0.328-1.434)$ & 0.316 \\
\hline MLL-PTD (mutated vs. wild) & $0.891(0.324-2.445)$ & 0.822 & $0.945(0.344-2.596)$ & 0.913 \\
\hline IDH1/IDH2 (mutated vs. wild) & $0.973(0.271-1.273)$ & 0.926 & $0.988(0.550-1.777)$ & 0.969 \\
\hline NRAS/KRAS (mutated vs. wild) & $1.214(0.637-2.314)$ & 0.556 & $1.228(0.644-2.340)$ & 0.532 \\
\hline \multicolumn{5}{|l|}{ Multivariate analyses } \\
\hline MiR-363 (high vs. low) & $2.362(1.346-4.145)$ & 0.003 & $2.683(1.507-4.779)$ & 0.001 \\
\hline WBC $\left(\geq 20\right.$ vs. $\left.<20 \times 10^{9} / L\right)$ & $1.806(1.036-3.151)$ & 0.037 & $1.861(1.056-3.280)$ & 0.032 \\
\hline RUNX1 (mutated vs. wild) & $1.706(0.797-3.654)$ & 0.169 & $1.819(0.850-3.892)$ & 0.123 \\
\hline TP53 (mutated vs. wild) & $2.786(1.312-5.915)$ & 0.008 & $2.566(1.221-5.395)$ & 0.013 \\
\hline
\end{tabular}

EFS event-free survival, OS overall survival, WBC white blood cell

Table 3 Univariate and multivariate analyses in patients treated with allo-HSCT

\begin{tabular}{|c|c|c|c|c|}
\hline \multirow[t]{2}{*}{ Variables } & \multicolumn{2}{|l|}{ EFS } & \multicolumn{2}{|l|}{ OS } \\
\hline & $\mathrm{HR}(95 \% \mathrm{CI})$ & $P$-value & $\mathrm{HR}(95 \% \mathrm{Cl})$ & $P$-value \\
\hline \multicolumn{5}{|l|}{ Univariate analyses } \\
\hline MiR-363 (high vs. low) & $1.182(0.643-2.175)$ & 0.590 & $1.424(0.775-2.619)$ & 0.255 \\
\hline WBC $\left(\geq 20\right.$ vs. $\left.<20 \times 10^{9} / L\right)$ & 1.089 (0.594-1.999) & 0.782 & $0.826(0.450-1.516)$ & 0.537 \\
\hline FLT3-ITD (positive vs. negative) & $1.876(0.914-3.851)$ & 0.086 & $1.973(0.953-4.084)$ & 0.067 \\
\hline NPM1 (mutated vs. wild) & 1.007 (0.515-1.970) & 0.983 & $1.023(0.523-1.998)$ & 0.948 \\
\hline DNMT3A (mutated vs. wild) & $1.285(0.655-2.520)$ & 0.466 & $1.387(0.704-2.731)$ & 0.344 \\
\hline RUNX1 (mutated vs. wild) & $1.145(0.449-2.290)$ & 0.777 & $1.579(0.613-4.067)$ & 0.344 \\
\hline TP53 (mutated vs. wild) & $2.034(0.718-5.760)$ & 0.181 & $4.334(1.453-12.925)$ & 0.009 \\
\hline TET2 (mutated vs. wild) & $0.526(0.127-2.186)$ & 0.377 & $0.670(0.162-2.776)$ & 0.581 \\
\hline MLL-PTD (mutated vs. wild) & $5.775(1.664-20.042)$ & 0.006 & $2.728(0.832-8.944)$ & 0.098 \\
\hline IDH1/IDH2 (mutated vs. wild) & $0.587(0.271-1.273)$ & 0.177 & $0.633(0.293-1.368)$ & 0.245 \\
\hline NRAS/KRAS (mutated vs. wild) & $0.796(0.245-2.586)$ & 0.705 & $0.488(0.150-1.587)$ & 0.233 \\
\hline \multicolumn{5}{|l|}{ Multivariate analyses } \\
\hline MLL-PTD (mutated vs. wild) & $5.180(1.449-18.511)$ & 0.011 & $3.136(0.943-10.429)$ & 0.062 \\
\hline FLT3-ITD (positive vs. negative) & $1.837(0.868-3.888)$ & 0.112 & $2.301(1.090-4.860)$ & 0.029 \\
\hline TP53 (mutated vs. wild) & $2.493(0.860-7.226)$ & 0.092 & $5.848(1.885-18.142)$ & 0.002 \\
\hline
\end{tabular}

EFS event-free survival, OS overall survival, WBC white blood cell

miR-363 expression, including 130 and 48 with positive and negative correlations, respectively (Fig. 3). Differentially upregulated genes in patients with high miR-363 expression included leukemogenic transcription factors (Myb, RUNX3, GATA3, IKZF3, HMGA2 and ETS1) [10, 11]. Notably, MLLT3/AF9 was up-regulated in the high
miR-363 group, as a frequent fusion partner of the MLL gene in translocations $\mathrm{t}(9 ; 11)(\mathrm{p} 22 ; \mathrm{q} 23)$ related to AML [12]. Among downregulated genes, we found that miR363 expression showed negative correlations with the levels of tumor suppressor genes (EZH2, KLF6 and PTEN). Interestingly, these three genes were predicted miR-363 

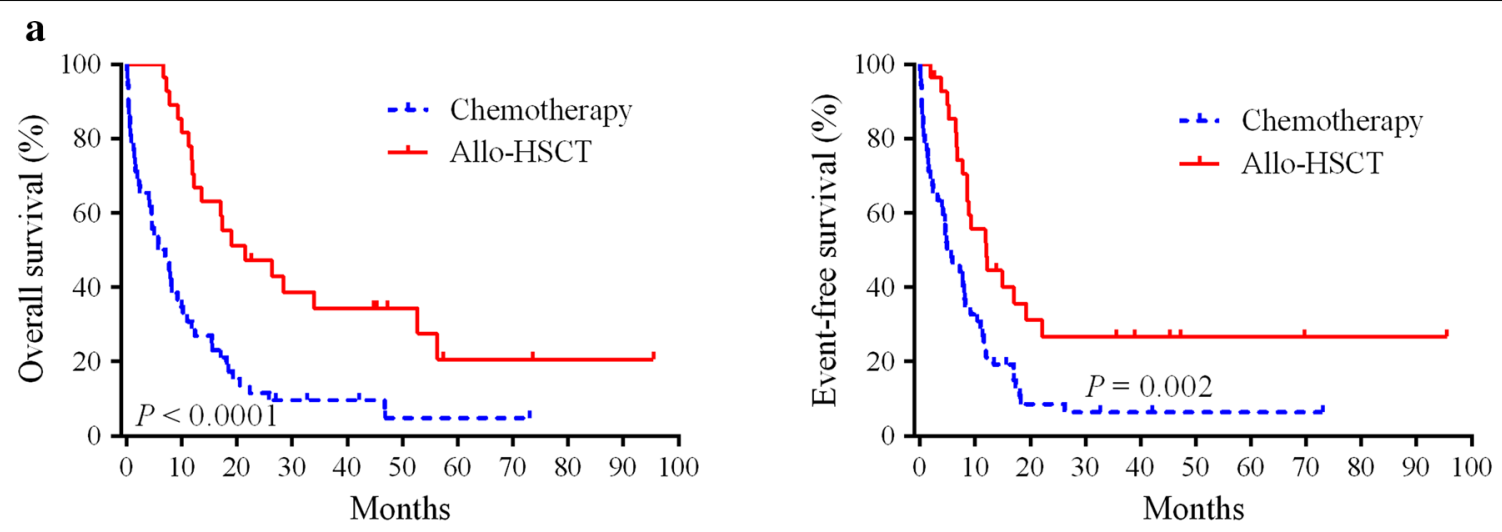

High miR-363 expression group

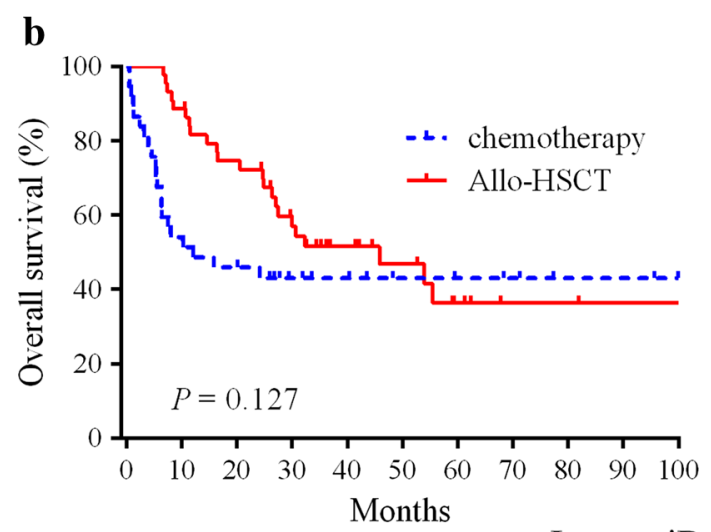

\section{Low miR-363 expression group}

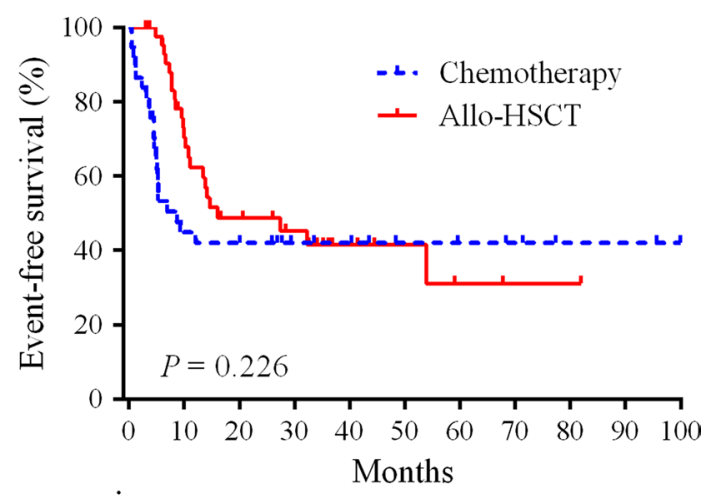

Fig. 2 Allo-HSCT overcomes the adverse prognostic influence of high miR-363 expression in AML. a The 162 cases were divided into two groups according to median miR-363 levels. Kaplan-Meier survival curves for cases administered chemotherapy $(n=53)$ and allo-HSCT $(n=28)$, respectively, in the high miR-363 group. b Kaplan-Meier survival curves for cases administered chemotherapy $(n=37)$ and allo-HSCT $(n=44)$, respectively, in the low miR-363 group

targets according to in silico analysis. Gene Ontology showed that genes associated with cell migration, $\mathrm{T}$ cell activation, system development, cell differentiation, response to chemicals and immune response were significantly correlated with miR-363 expression (Table 4). Thus, the miR-363 associated gene-expression profiling signature supported clinical finding in AML obtained by miRNA analysis.

\section{Discussion}

As current molecular stratification schemes do not fully grasp the heterogeneity of prognosis in patients with AML, the identification of novel prognostic markers is urgent [13]. In heterogeneous cohorts of AML patients, the correlation of miRNAs as predictive molecular markers remains largely unknown. In this study, miR363 was determined as an independent prognostic factor in AML cases undergoing chemotherapy. Meanwhile, the miR-363 expression provides a powerful tool for risk stratification of AML patients. More importantly, allo-HSCT can overcome miR-363 expressionassociated adverse outcomes.

We showed that miR-363 expression levels constitute independent prognostic marker of AML in a heterogeneous cohort administered chemotherapy. High miR-363 levels could still predict adverse outcome after consideration of other molecular prognostic factors in multivariable analysis. Thus, miR-363 could increase the prognostic value of previously defined molecular factors in a highly heterogeneous cohort of AML cases. Strikingly, patients with high miR-363 expression levels showed markedly poor OS and EFS. These findings suggest that miR-363 independently influences treatment outcomes and may synergistically drive leukemogenesis. More importantly, miR-363 expression levels could be useful to the identification of patients with adverse outcome in AML patients administrated chemotherapy. 


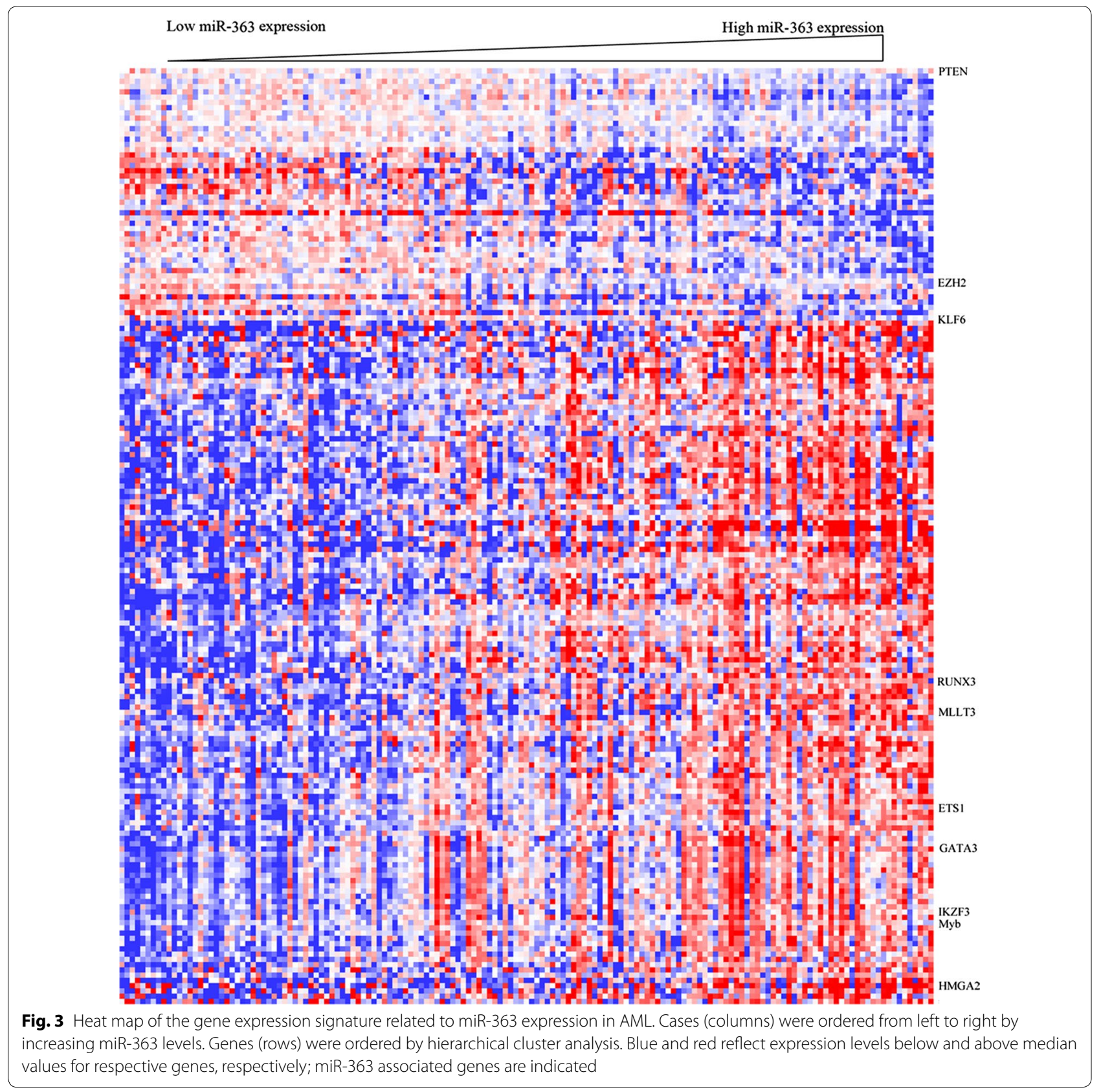

Conventional chemotherapy and allo-HCST constitute the standard post-remission treatment strategies for AML [14]. However, there is a lack of efficient prognostic markers for guiding rational treatment options. We found that high miR-363 expressers administered allo-HSCT showed markedly improved OS and EFS in comparison with cases administered chemotherapy. In cases lowly expressing miR-363, there was no advantage for those administered allo-HSCT in comparison with the chemotherapy group. These findings indicate that patients with low miR-363 expression may not benefit from allo-HSCT as first-line therapy. Therefore, the expression of miR-363 may contribute to identify patients in need of strategies to select the optimal treatment regimen between chemotherapy and allo-HCST. The AML patients with high miR-363 expression may be preferably recommended for early allo-HSCT.

The possible oncogenic function of miR-363 has been reported previously in T-cell acute lymphoblastic leukemia, multiple myeloma and solid tumors $[15,16]$. MiR363 promotes growth and chemo-resistance in gastric adenocarcinoma by downregulating FBW7 [17]. It was 
Table 4 Gene ontology terms of biological processes in the miR-363 associated expression profile

\begin{tabular}{|c|c|c|c|}
\hline GO ID & GO terms & $\begin{array}{l}\text { Percentage of members of the } \mathrm{GO} \text { term } \\
\text { present in the miR- } 363 \text { profile }\end{array}$ & $P$-value \\
\hline GO:0050789 & Regulation of biological process & 69.5 & $<0.001$ \\
\hline GO:0050794 & Regulation of cellular process & 64.4 & $<0.001$ \\
\hline GO:0051716 & Cellular response to stimulus & 44.1 & 0.009 \\
\hline GO:0007154 & Cell communication & 40.7 & 0.009 \\
\hline GO:0032502 & Developmental process & 39.0 & 0.002 \\
\hline GO:0007275 & Multicellular organism development & 37.3 & $<0.001$ \\
\hline GO:0048731 & System development & 33.9 & $<0.001$ \\
\hline GO:0048869 & Cellular developmental process & 29.4 & 0.003 \\
\hline GO:0030154 & Cell differentiation & 28.2 & 0.001 \\
\hline GO:0042221 & Response to chemical & 27.7 & 0.025 \\
\hline GO:0010033 & Response to organic substance & 20.3 & 0.026 \\
\hline GO:0070887 & Cellular response to chemical stimulus & 19.8 & 0.018 \\
\hline GO:0009605 & Response to external stimulus & 16.9 & 0.009 \\
\hline GO:0045595 & Regulation of cell differentiation & 15.3 & 0.001 \\
\hline GO:0006955 & Immune response & 13.6 & 0.012 \\
\hline
\end{tabular}

GO gene ontology

shown that miR-363 is a prognostic marker for hepatocellular carcinoma [18]. However, the function and prognostic role of miR-363 in AML remains unclear. To derive biological insights from AML cases characterized by high miR-363 expression, we identified genes associated with miR-363 expression in AML patients. Interestingly, miR-363 expression was positively correlated with the amounts of leukemogenic transcription factors, including Myb, RUNX3, GATA3, IKZF3, ETS1 and MLLT3. The Myb oncogene, a driver of leukemogenesis, is widely expressed in AML and important for continued proliferation and differentiation blocking activity in AML cells [19]. ETS1 is critical in cell proliferation and differentiation in AML [20]. MLLT3 represents a commonly encountered fusion partner of MLL in translocations $\mathrm{t}(9 ; 11)(\mathrm{p} 22 ; \mathrm{q} 23)$, which are related to AML [21]. Notably, we found that the direct target genes (EZH2, KLF6 and PTEN) of miR-363 were downregulated in association with high miR-363 expression. It was shown that loss-of-function mutations of the tumor suppressor gene EZH2 are found in AML [22]. Meanwhile, PTEN plays an essential role in the prevention of leukemogenesis [23, 24]. Indeed, PTEN deletion in hematopoietic cells can induce a myeloproliferative disease within days and transplantable leukemias within weeks. These miR-363 associated genes may participate in the adverse response to chemotherapy in cases highly expressing miR-363. Therefore, the miR-363 related gene-expression profiling signature may support the clinical observation that AML is characterized by the expression of miRNA. However, the mechanisms concerning the regulation of miR-363 expression and subsequent influence of AML treatment outcome remain to be elucidated.

\section{Conclusions}

In conclusion, miR-363 levels independently correlate with clinical outcome in a highly heterogeneous cohort of AML cases. MiR-363 expression could greatly contribute to the identification of patients with poor outcome in AML. Expression analysis of miR-363 may be useful to improve the risk stratification of AML patients. Furthermore, allo-HSCT may overcome the unfavorable consequences of high miR-363 expression in AML. Therefore, the expression analysis of miR-363 may help identify cases in need of strategies to select the optimal treatment regimen between chemotherapy and allo-HCST.

\footnotetext{
Abbreviations

AML: acute myeloid leukemia; Allo-HSCT: allogeneic hematopoietic stem cell transplantation; TCGA: The Cancer Genome Atlas; OS: overall survival; EFS: event-free survival.

Authors' contributions

$\mathrm{HZ}, \mathrm{NZ}$ and RW designed and performed the computational analyses. TS, YF, YY, QW, JC, SZ, HZ and ZL contributed to statistical analyses. $\mathrm{XL}, \mathrm{MN}$ and $\mathrm{KX}$ designed and wrote the manuscript. All authors read and approved the final manuscript.

\section{Author details}

1 Blood Diseases Institute, Xuzhou Medical University, Xuzhou, Jiangsu, China. ${ }^{2}$ Department of Hematology, Affiliated Hospital of Xuzhou Medical University, Xuzhou, Jiangsu, China. ${ }^{3}$ Jiangsu Key Laboratory of Bone Marrow Stem Cell, Xuzhou Medical University, Xuzhou, Jiangsu, China. ${ }^{4}$ Insititute of Nervous System Diseases, Xuzhou Medical University, Xuzhou, Jiangsu, China.
}

\section{Acknowledgements}

The authors thank The Cancer Genome Atlas Network. 


\section{Competing interests}

The authors declare that they have no competing interests.

\section{Availability of data and materials}

The datasets supporting the conclusions of this article are included within this article. Clinical data for all patients, including the treatment approach and outcomes data, are publicly accessible from the TCGA website (https://cance rgenome.nih.gov).

\section{Consent for publication}

Not applicable.

\section{Ethics approval and consent to participate}

Not applicable.

\section{Funding}

The research was supported by National Natural Science Foundation of China (81670142, 81772658, 81870163, 81700179); Jiangsu Provincial Key Research and Development Program (BE2017638, BE2017636); Natural Science Foundation of Jiangsu Province (BK20180104, BK20160226); The Foundation of Jiangsu Province Six Talents Peak (2017-WSN-120); Jiangsu Qing Lan Project for Mingshan Niu; Xuzhou Key Research and Application of Basic Research Projects (KC17158); Postgraduate Research \& Practice Innovation Program of Jiangsu Province (SJCX18_0704, SJKY19_2110).

\section{Publisher's Note}

Springer Nature remains neutral with regard to jurisdictional claims in published maps and institutional affiliations.

Received: 14 January 2019 Accepted: 26 March 2019

Published online: 01 April 2019

\section{References}

1. Paguirigan AL, Smith J, Meshinchi S, Carroll M, Maley C, Radich JP. Singlecell genotyping demonstrates complex clonal diversity in acute myeloid leukemia. Sci Transl Med. 2015;7:281 re282.

2. Dohner H, Estey E, Grimwade D, Amadori S, Appelbaum FR, Buchner T, et al. Diagnosis and management of AML in adults: 2017 ELN recommendations from an international expert panel. Blood. 2017;129:424-47.

3. Papaemmanuil E, Gerstung M, Bullinger L, Gaidzik VI, Paschka P, Roberts ND, et al. Genomic classification and prognosis in acute myeloid leukemia. N Engl J Med. 2016;374:2209-21.

4. Yeh $\mathrm{CH}$, Moles $\mathrm{R}$, Nicot C. Clinical significance of microRNAs in chronic and acute human leukemia. Mol Cancer. 2016;15:37.

5. Yang C, Shao T, Zhang H, Zhang N, Shi X, Liu X, et al. MiR-425 expression profiling in acute myeloid leukemia might guide the treatment choice between allogeneic transplantation and chemotherapy. J Transl Med. 2018;16(1):267.

6. Schwind S, Maharry K, Radmacher MD, Mrozek K, Holland KB, Margeson $D$, et al. Prognostic significance of expression of a single microRNA, miR181a, in cytogenetically normal acute myeloid leukemia: a Cancer and Leukemia Group B study. J Clin Oncol. 2010;28:5257-64.

7. Sun SM, Rockova V, Bullinger L, Dijkstra MK, Dohner H, Lowenberg B, et al. The prognostic relevance of miR-212 expression with survival in cytogenetically and molecularly heterogeneous AML. Leukemia. 2013;27:100-6.
8. Eisfeld AK, Marcucci G, Maharry K, Schwind S, Radmacher MD, Nicolet D et al. miR-3151 interplays with its host gene BAALC and independently affects outcome of patients with cytogenetically normal acute myeloid leukemia. Blood. 2012;120:249-58.

9. Cancer Genome Atlas Research N, Ley TJ, Miller C, Ding L, Raphael BJ, Mungall AJ, et al. Genomic and epigenomic landscapes of adult de novo acute myeloid leukemia. N Engl J Med. 2013;368:2059-74.

10. Pattabiraman DR, Gonda TJ. Role and potential for therapeutic targeting of MYB in leukemia. Leukemia. 2013:27:269-77.

11. Stavast CJ, Leenen PJM, Erkeland SJ. The interplay between critical transcription factors and microRNAs in the control of normal and malignant myelopoiesis. Cancer Lett. 2018;427:28-37.

12. Stavropoulou V, Kaspar S, Brault L, Sanders MA, Juge S, Morettini S, et al. MLL-AF9 expression in hematopoietic stem cells drives a highly invasive AML expressing EMT-related genes linked to poor outcome. Cancer Cell. 2016;30:43-58

13. KIco JM, Spencer DH, Miller CA, Griffith M, Lamprecht TL, O'Laughlin M, et al. Functional heterogeneity of genetically defined subclones in acute myeloid leukemia. Cancer Cell. 2014;25:379-92.

14. Cornelissen JJ, Versluis J, Passweg JR, van Putten WL, Manz MG, Maertens J, et al. Comparative therapeutic value of post-remission approaches in patients with acute myeloid leukemia aged 40-60 years. Leukemia. 2015;29:1041-50

15. Landais S, Landry S, Legault P, Rassart E. Oncogenic potential of the miR106-363 cluster and its implication in human T-cell leukemia. Cancer Res. 2007;67:5699-707.

16. Gowda PS, Wildman BJ, Trotter TN, Xu X, Hao X, Hassan MQ, et al. Runx2 suppression by miR-342 and miR-363 inhibits multiple myeloma progression. Mol Cancer Res. 2018;16:1138-48.

17. Zhang PF, Sheng LL, Wang G, Tian M, Zhu LY, Zhang R, et al. miR363 promotes proliferation and chemo-resistance of human gastric cancer via targeting of FBW7 ubiquitin ligase expression. Oncotarget. 2016;7:35284-92.

18. Zhang J, Fan J, Zhou C, Qi Y. miR-363-5p as potential prognostic marker for hepatocellular carcinoma indicated by weighted co-expression network analysis of miRNAs and mRNA. BMC Gastroenterol. 2017;17:81.

19. Pattabiraman DR, McGirr C, Shakhbazov K, Barbier V, Krishnan K, Mukhopadhyay $P$, et al. Interaction of c-Myb with p300 is required for the induction of acute myeloid leukemia (AML) by human AML oncogenes. Blood. 2014;123:2682-90.

20. Lulli V, Romania P, Riccioni R, Boe A, Lo-Coco F, Testa U, et al. Transcriptional silencing of the ETS1 oncogene contributes to human granulocytic differentiation. Haematologica. 2010;95:1633-41.

21. Ney Garcia DR, Liehr T, Emerenciano M, Meyer C, Marschalek R, Pombode-Oliveira Mdo S, et al. Molecular studies reveal a MLL-MLLT3 gene fusion displaced in a case of childhood acute lymphoblastic leukemia with complex karyotype. Cancer Genet. 2015;208:143-7.

22. Ernst T, Chase AJ, Score J, Hidalgo-Curtis CE, Bryant C, Jones AV, et al. Inactivating mutations of the histone methyltransferase gene $\mathrm{EZH} 2$ in myeloid disorders. Nat Genet. 2010;42:722-6.

23. Yilmaz OH, Valdez R, Theisen BK, Guo W, Ferguson DO, Wu H, et al. Pten dependence distinguishes haematopoietic stem cells from leukaemiainitiating cells. Nature. 2006:441:475-82.

24. Zhang J, Grindley JC, Yin T, Jayasinghe S, He XC, Ross JT, et al. PTEN maintains haematopoietic stem cells and acts in lineage choice and leukaemia prevention. Nature. 2006;441:518-22. 